Review Article

\title{
Exploring pathophysiology of COVID-19 infection: Faux espoir and dormant therapeutic options
}

\author{
Vinod Nikhra* \\ Senior Consultant and Faculty, Department of Medicine, Hindu Rao Hospital and NDMC Medical \\ College, New Delhi, India
}

\section{Abstract}

COVID-19 virus structural components: The 2019-nCoV, also called SARS-CoV-2, was first reported in Wuhan, China in December 2019. The disease was named Coronavirus Disease 2019 (COVID-19) and the virus responsible for it as the COVID-19 virus, respectively, by WHO. The 2019-nCoV has a round, elliptic or pleomorphic form with a diameter of 60-140 nm. It has single-stranded RNA genome containing 29891 nucleotides, a lipid shell, and spike, envelope, membrane and hemagglutinin-esterase (HE) proteins.

Steps in progression of COVID-19 illness: Once inside the airways, the S protein on the viral surface recognizes and mediates the attachment to host ACE-2 receptors and gains access to endoplasmic reticulum. The HE protein facilitates the $S$ protein-mediated cell entry and virus spread through the mucosa, helping the virus to attack the ACE2-bearing cells lining the airways and infecting upper as well as lower respiratory tracts. With the dying cells sloughing down and filling the airways, the virus is carried deeper into the lungs. In addition, the virus is able to infect ACE2-bearing cells in other organs, including the blood vessels, gut and kidneys. With the viral infestation, the activated immune system leads to inflammation, pyrexia and pulmonary edema. The hyperactivated immune response, called cytokine storm in extreme cases, can damage various organs apart from lungs and increases susceptibility to infectious bacteria especially in those suffering from chronic diseases.

The current therapeutics for COVID-19: At present, there is no specific antiviral treatment available for the disease. The milder cases may need no treatment. In moderate to severe cases, the clinical management includes infection prevention and control measures, and symptomatic and supportive care, including supplementary oxygen therapy. In the critically ill patients, mechanical ventilation is required for respiratory failure and hemodynamic support is imperative for managing circulatory failure and septic shock.

Conclusion: Confusion, despair and hopes: There is no vaccine for preexposure prophylaxis or postexposure management. There are no specific approved drugs for the treatment for the disease. A number of drugs approved for other conditions as well as several investigational drugs are being canned and studied in several clinical trials for their likely role in COVID-19 prophylaxis or treatment. The future seems afflicted with dormant therapeutic options as well as faux Espoir or false hopes. As obvious, not all clinical trials will be successful, but having so many efforts in progress, some may succeed and provide a positive solution. Right now, though, confusion and despair prevail.

\section{Exploring pathophysiology of COVID-19}

Witnessing and defining the disease: The coronaviruses (CoVs) are positive-stranded RNA viruses with a crown-like appearance due to the presence of large spike glycoproteins on the envelope. There are seven CoVs capable of infecting humans (HCoVs), out of which HCoV-OC43 and HCoV-HKU1 (betaCoVs of the A lineage), and HCoV-229E and HCoV-NL63 (alphaCoVs) were identified in the 1960s and responsible for about $5 \%$ to $10 \%$ cases of common cold and short-term upper respiratory infections, with about $2 \%$ of the human population being healthy carriers of a $\mathrm{CoV}$. The remaining three HCoVs belonging to betaCoVs of the $\mathrm{B}$ and $\mathrm{C}$ lineage, have been discovered only in recent years and include SARS-CoV,

\section{More Information}

*Address for Correspondence: Dr. Vinod Nikhra, M.D., Consultant and Faculty, Department of Medicine, Hindu Rao Hospital \& NDMC Medical College, New Delhi, India, Tel: 91-9810874937; Email: drvinodnikhra@gmail.com; drvinodnikhra@rediffmail.com

Submitted: 14 April 2020 Approved: 05 May 2020 Published: 06 May 2020

How to cite this article: Nikhra V. Exploring pathophysiology of COVID-19 infection: faux espoir and dormant therapeutic options. Int $\mathrm{J}$ Clin Virol. 2020; 4: 034-040.

DOI: 10.29328/journal.ijcv.1001013

\section{ORCiD: orcid.org/0000-0003-0859-5232}

Copyright: @ 2020 Nikhra V. This is an open access article distributed under the Creative Commons Attribution License, which permits unrestricted use, distribution, and reproduction in any medium, provided the original work is properly cited.

Keywords: COVID-19; 2019 nCoV; Spike (S) protein; ACE-2; Cytokine storm syndrome; Azithromycin; Chloroquine; Hydroxychloroquine; Remdesivir; COVID-19 vaccine

Check for updates

OPEN ACCESS 
COVID-19 virus structural components: The SARSCoV-2 or 2019-nCoV has a round, elliptic or pleomorphic form with a diameter of $60-140 \mathrm{~nm}$. Its single-stranded RNA genome contains 29891 nucleotides, encoding for 9860 amino acids and bears a sequence identity approximately $50 \%$ to MERS-CoV and 79\% to SARS-CoV [2]. Like other CoVs, it is sensitive to ultraviolet rays and heat and can be effectively inactivated by lipid solvents including ether and ethanol.

The 2019-nCoV has a lipid shell, an RNA genome, spike, envelope and membrane and hemagglutinin-esterase dimer proteins (Figure 1). The world-wide research is going on to determine the structural components of the $2019-\mathrm{nCoV}$ as related to the pathogenetic mechanisms [3]. The glycosylated Spike protein (S) is a large component making the distinct spikes on the surface of the virus. It utilizes an $\mathrm{N}$-terminal signal sequence to gain access to the endoplasmic reticulum and mediates the attachment to host angiotensin-converting enzyme II (ACE2) receptors. The S protein is cleaved by a host cell furin-like protease into two separate polypeptides $\mathrm{S} 1$ and S2.The RNA genome of the virus is bound by the phosphorylated nucleocapsid protein $(\mathrm{N})$ in a beads-on-astring conformation. On entering the host cells, the $\mathrm{N}$ protein potentially tethers the viral genome to replicase-transcriptase complex (RTC), and helps in packaging the encapsulated genome into viral particles [4].

The Envelope protein (E) is found in small quantities in the virus and is appears to be a transmembrane protein with ion channel activity. The protein facilitates assembly and release of the new virions. It is related to the disease pathogenesis and important for the disease progression. The Membrane protein (M) is the most abundant structural component of the virus. It exists as a dimer and enables to maintain membrane curvature on one end and bound to nucleocapsid proteins on the other.

The Hemagglutinin-esterase (HE) is also a dimer protein and binds to sialic acids on surface glycoproteins. It is responsible for facilitating and enhanceing $S$ protein-mediated cell entry and virus spread through the mucosa.

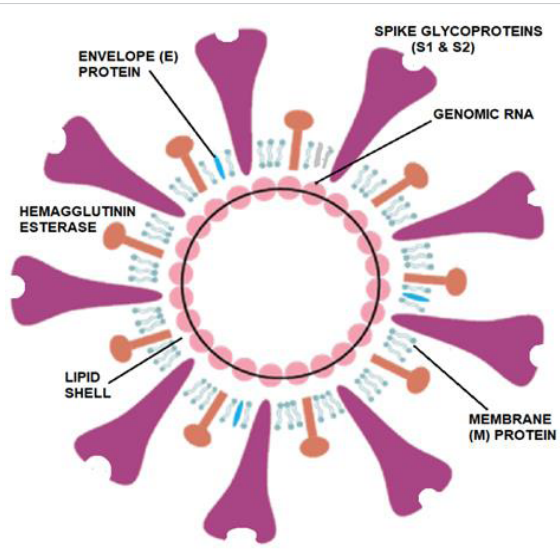

Figure 1: The structural diagram and components of COVID-19 Virus. Modified from: https://d1gk4lfg3bst7c.cloudfront.net/media/wysiwyg/2019-nCoV-figure-ad.png
The viral transmission and evaluation: As with other respiratory pathogens, including flu and rhinovirus, the transmission is believed to occur through respiratory droplets from coughing and sneezing or by being carried to oral or nasal mucosa by hands from the virus-infested surfaces. It appears that all COVID-19 patients - asymptomatic, mild or severe have a massive throat/mucus titre of virus, shedding it in the surroundings. Aerosol transmission may also be possible in closed and confined spaces. The incubation period is from 3 to 7 days, 14 days being considered as the longest possible time from infection to appearance of symptoms [5]. The research supports that in addition to the respiratory droplets and direct contact, fecal-oral transmission might also be the route of transmission of 2019-nCoV. It is to be noted that the information is derived from the early research and reports. Further studies are warranted to understand the mechanisms of transmission, the incubation period, duration of infectivity and the clinical course of COVID-19 [6].

The WHO recommends collecting specimens from both the upper respiratory tract (nasopharyngeal and oropharyngeal samples) and lower respiratory tract such as expectorated sputum, endotracheal aspirate, or bronchoalveolar lavage for a diagnostic test. The samples are needed to be stored at $4^{*} \mathrm{C}$. The amplification of the genetic material extracted from the sample is done through a reverse polymerase chain reaction (RT-PCR). If the test result is positive, it is recommended that the test is to be repeated for verification. In patients with confirmed COVID-19 diagnosis, the laboratory evaluation should be repeated at intervals to evaluate for viral clearance prior to discharge from observation. In a positive case, lymphopenia as well as raised liver enzymes, LDH, muscle enzymes and C-reactive are negative prognostic factors.

Steps in progression of COVID-19 illness: Once inside the airways, the $\mathrm{S}$ protein on the viral surface recognize and stick to the receptor protein called ACE2 and the virus attacks the ACE2-bearing cells lining the airways. It can infect upper as well as lower respiratory tracts and with the dying cells sloughing down and filling the airways the virus is carried deeper into the lungs. The thin layer of surfactant coating the airways becomes even thinner and the brush border less efficient to evict viruses and other foreign particles with a colder temperature and dry air, which may also dampen the immune response to the invading viruses. It appears that the virus is able to transmits while still confined to the upper airways, before invading the lower respiratory tract and lungs and causing severe symptoms. In addition, the virus is able to infect ACE2-bearing cells in other organs, including the blood vessels, gut and kidneys.

With the viral infestation, the activated immune system leads to macrophages to be recruited to the alveolar space, which increase cytokine production and attract additional immune cells to the affected area such as T-helper cells CD4 and CD8 to combat the virus. The pattern recognition receptors 
(PRRs) of the immune cells recognize the virus and signal release of the pro-inflammatory cytokines such as interferon gamma (IFN-g), tumor necrosis factors (TNFs), interleukins (ILs), and chemokines. IFN-g activates macrophages which produce IL-6, TNF- $\alpha$, and IL-10. There are triggered additional pathways associated with PRRs, including cyclo-oxygenase (COX)-2 and c-Jun N-terminal kinase (JNK) [7]. With recovery, once the virus is cleared, the immune pathways shut down. In a cytokine storm, however, the process goes into overdrive, initiating vascular leakage, coagulation cascades, and disseminated intravascular coagulation (DIC). Further, it leads to an increased susceptibility to infectious bacteria. Furthermore, the process affect other organs besides the lungs, especially in those suffering chronic diseases, leading to multi-organ failure (MOF) [8].

Age is an important epidemiological factor. The elderly people are at risk of severe infections possibly because the ineffective initial anti-viral immune response. It appears that children may be less severely affected because their immune system is unlikely to progress to a cytokine storm. Further, there are other factors like individual genetic make-up, the amount of virus load, the other microbes in the body including gut microbiota which may play a role in acquirement of the infection and its progression.

Directed by the viral RNA, the synthesis of polyprotein 1a/1ab (pp1a/pp1ab) in the host cells is brought about with the transcription occurring through the replication-transcription (RT) complex organized in double-membrane vesicles (Figure 2 ). The open reading frames (ORFs) guide the production of both pp1a and pp1ab polypeptides which are processed by virally encoded chymotrypsin-like protease (3CLpro) or main protease (Mpro), as well as one or more papain-like proteases for producing 16 non-structural proteins (nsps) [9]. The ORFs also encode for structural proteins, including spike, membrane, envelope and nucleocapsid proteins. The pathophysiology and virulence mechanisms of COVID-19 virus has been related to the function of the nsps and structural proteins. It has been documented that the nsps are able to block the host innate immune response, as well [10]. The E protein also plays a crucial role in in promoting viral assembly and release of nascent virions.

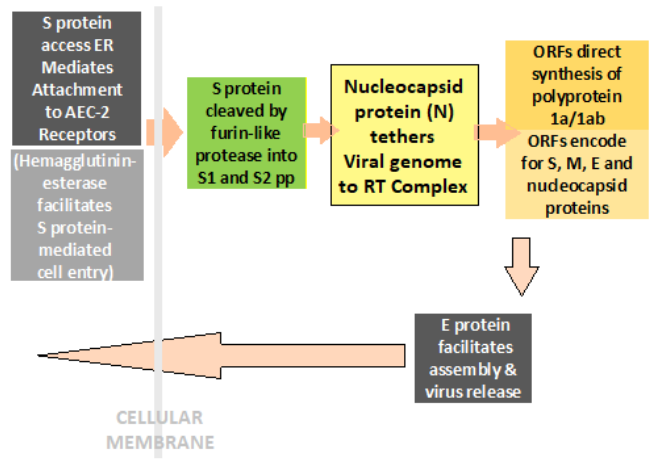

Figure 2: The Simplified Steps in Pathogenesis and Replication Cycle of COVID-19.
Ace2 receptors and expression in COVID-19: It appears that ACE2 is the main host cell receptors for 2019-nCoV, playing a crucial role in the invasion of virus into the cell through the receptor binding domain (RBD) and infectivity and affliction of upper respiratory tract, lower respiratory tract and lungs, and gastrointestinal system apart from kidneys and various other organs [11]. Other studies have indicated that the ACE2 is the major cell receptors for 2019$\mathrm{nCoV}$ and the 2019-nCoV may not preferentially use other receptors like aminopeptidase $\mathrm{N}$ and dipeptidyl peptidase 4 (DPP4). Further, the $2019 \mathrm{nCoV}$ spike (S) protein plays the most important roles in viral attachment, fusion and entry [12]. Considering this, the expression and distribution of the ACE2 in human body may outline the potential infection routes and the organs with high ACE2-expressing tissues are potential sites for 2019-nCoV infection [13]. Further, the ACE2 expression is reduced in SARS-CoV infection and linked with the viral S protein. In mice experiments, the injection of SARS$\mathrm{CoV}$ Spike worsens acute lung failure in vivo, which can be attenuated by blocking the renin-angiotensin pathway [14].

Significantly high ACE2 expression is found in type II alveolar cells (AT2) of lung, upper oesophageal stratified epithelial cells, absorptive enterocytes from ileum and colon, gall bladder and bile ductal cells, myocardial cells, kidney proximal tubule cells and bladder urothelial cells. Apart from this, the ACE2 is also expressed on the oral mucosa and highly enriched in epithelial cells of tongue accounting for a potentially high risk of this route for 2019-nCoV infectious susceptibility [15]. As such, the ACE2 expression is higher in oral mucosa and tongue than buccal and gingival tissues. These findings may underline the prophylactic role of frequent mouth washing and rinsing [16]. The researchers have isolated 2019-nCoV from human airway epithelial cells. Further, the saliva, urine and stool specimens and rectal swabs have demonstrated embedded viruses in the COVID-19 patients [17].

It is worth mentioning that bulk of the ACE2-expressing cells, over $80 \%$, are AT2 (alveolar type 2) cells. In addition, the distribution of ACE2 is more widespread in males than females, which is consistent with the epidemiological finding that men are more prone to Covid-19 than women. The analysis of the ACE2 RNA expression profile indicates that the ACE2 expressing of ACE2 AT2 cells also express many other genes favouring the viral processes. The abundant expression of ACE2 in the AT2 cells may explain the preferential involvement of lungs and severe alveolar damage following the infection. It has also been noticed that the Asian men have a relatively higher ACE2- expressing cell ratio than the Caucasian and African American subjects [18]. But, these reports of the ACE2 expression analysis in lung tissues are controversial, in light of small numbers of subjects. In another study, the ACE2 expression analysis using the RNA-seq and microarray datasets from control lung tissues indicated there were no significant differences between Asian and Caucasian, or male and female [19]. 


\section{Current and dormant therapeutic possibilities}

Clinical spectrum of COVID-19: The clinical spectrum of COVID-19 varies from asymptomatic or pauci-symptomatic presentation to a moderate to severe states characterized by respiratory failure necessitating mechanical ventilation and ICU support and those manifesting critical clinical conditions like sepsis, septic shock and multiple organ dysfunction syndromes (MODS). The mild disease, i.e., non-pneumonia and mild pneumonia occurs approximately in $81 \%$ of cases. A severe disease with dyspnoea and acute respiratory distress syndrome (ARDS) and/or lung infiltrates appearing within 24 to 48 hours occurs in about $14 \%$ of cases. Whereas, the critical COVID-19 illness accompanied by respiratory failure, septic shock, and/or multiple organ dysfunction (MOD) or failure (MOF) is likely to occur approximately in $5 \%$ of cases [20].

The transition from milder symptoms to acute respiratory distress syndrome (ARDS) is likely due to an unrestrained cytokine release by the hyperactive immune response. It appears that in patients who are going to have the worst outcomes with COVID-19 infections, the immune system becomes overactive with excessive production of $\mathrm{T}$ cells and macrophages, resulting in cytokine storm with release of a large amount of proinflammatory cytokines including interleukins (IL) - 1, 6, 12 and 18. The excessive or uncontrolled levels of cytokines are released then further activate more immune cells, resulting in hyperinflammation and cytokine release syndrome (CRS).

The long-term complications among survivors of infection with SARS-CoV-2 having clinically significant COVID-19 disease are not yet available [21]. A note is to be taken of the clinical evidence suggesting that anosmia and dysgeusia associated with the COVID-19 infection. This may be linked to the rich ACE2 receptors expressed in nasal mucosa and tongue affected on the viral infection [22]. These subtle symptoms could in many cases be harbingers of a coronavirus diagnosis. Anosmia, in particular, has been seen in patients ultimately testing positive for the coronavirus with no other symptoms [23]. With the recovery from the disease, most patients gradually regain both their sense of taste and smell. The gastrointestinal symptoms in COVID-19 include anorexia, diarrhoea, nausea and vomiting, abdominal colic and, rarely, gastrointestinal bleeding. Diarrhoea is the most common abdominal symptom in children as well as adults in COVID-19 [24].

\section{Treatment and management}

There is no vaccine for preexposure prophylaxis or postexposure management. Simultaneously, there is no specific antiviral treatment recommended for COVID-19. The treatment is mostly symptomatic, and oxygen therapy and ventilatory support represents the major treatment options available for patients with severe infection. In the critically ill patients, assisted mechanical ventilation is required for respiratory failure and hemodynamic support is imperative for managing circulatory failure and septic shock [25].
Among other therapeutic modalities, the use of systemic corticosteroids for the treatment of viral pneumonia accompanied by ARDS is not recommended [26]. Further, routine or inappropriate administration of antibiotics is not recommended. Some studies have cited use of azithromycin along with chloroquine (CQ) or hydroxychloroquine (HCQ) has been tried. So far, although no antiviral treatments have been approved, alpha-interferon (e.g., 5 million units by aerosol inhalation twice per day), lopinavir/ritonavir and remdesivir have been suggested to be effective.

\section{Clinical/experimental trials}

The anti-viral drugs: There have been clinical trials using the combination of the HIV protease inhibitors Lopinavir (acts against the viral 3CL protease and has a modest antiviral activity against COVID-19) and Ritonavir, which increases the bioavailability of the former. The results from the trials were not found to be promising as no benefit has been documented in the primary end point of time to clinical improvement [27]. Though, there was shown slightly smaller number of deaths in the patient group treated with the lopinavir-ritonavir combination. Further, there was no significant effect on viral shedding. In another study in China, Kaletra (lopinavirritonavir combination) was not found to be a promising treatment of hospitalized COVID-19 patients with pneumonia in a recent clinical trial in China [28]. There has also been use of chloroquine along with lopinavir-ritonavir combination [29].

A combination of Oseltamivir and Lopinavir and ritonavir, the potent protease inhibitors, have been used to treat severe cases of 2019-nCoV. The team of Thai doctors in Rajavithi Hospital, Bangkok who have been caring for COVID-19 patients, used large doses of oseltamivir combined with HIV drugs lopinavir and ritonavir. Though not cured, the patient's condition reportedly improved during a 10 day follow up, with the test result becoming negative within 48 hours following administration of the drug combination [30].

Some studies have reported, Favipiravir (6-fluoro-3hydroxy-2-pyrazinecarboxamide), a selective RNA polymerase inhibitor, showing useful effects in COVID-19. Eighty patients suffering from COVID-19 participated in the clinical trial of which 35 patients took Favipiravir and 45 formed the control arm [31]. Improvement was noted in lungs on X-rays in about 91 percent patients given the medicine compared to $62 \%$ of those without it. In addition, they turned negative for the virus after a median of four days after becoming positive, compared with a median of 11 days for those who were not treated with the drug. It was concluded in the trial that ordinary COVID-19 patients untreated with antiviral previously, Favipiravir can be considered as a preferred treatment because of its higher recovery rate and reduced incidence of pyrexia and cough. There were few associated adverse effects [32].

The anti-viral drug, Remdesivir was found to be beneficial by inhibiting RNA replication in MERS and SARS patients. The 
drug acts as nucleotide analogues interfering with the RNAdependent RNA polymerase. In an in vitro study, Remdesivir blocked coronavirus infection in monkey and human cells. It is held that it may work against COVID-19, as it inhibits the coronavirus that causes MERS in cell culture and improves respiratory symptoms in the animal studies [33]. The drug inhibits viral replication through a premature termination of RNA transcription and has shown in vitro activity against SARS-CoV-2, and in vitro as well as in vivo activity against related beta-Coronaviruses. It appears to be particularly effective in combination with chloroquine [34].

Macrolide Antibiotic - Azithromycin: Azithromycin is a macrolide antibiotic and works by decreasing protein synthesis by bacteria by binding to the $50 \mathrm{~S}$ subunit of the bacterial ribosome and inhibitingtranslation ofmRNA.AFrench study has shown promising results for COVID-19 patients with hydroxychloroquine and azithromycin combination. It significantly reduced viral load [35]. The French study was not a controlled clinical trial. A number of researchers have advised that the evidence should be re-examined, and further studies/research are needed. Occasionally, azithromycin can causes cholestatic hepatitis or delirium, whereas its overdose may cause severe heart block and increase the risk of death, especially in patients with heart problems.

Aminoquinolines (4AQs) - CQ and HCQ: Both the drugs, CQ and HCQ, have shown in-vitro activity against SARS-CoV-2, with HCQ having comparatively relatively higher potency. A study in China has documented that CQ treatment of COVID-19 patients had clinical and virologic benefit versus a control group [36]. Based upon in-vitro studies and limited data, CQ or HCQ are being currently recommended for treatment of hospitalized COVID-19 patients in several countries [37]. Though CQ and HCQ, both, have known safety profiles, the main concern is about cardiotoxicity - prolongation of QT interval, risk of hepatic and renal dysfunction with prolonged use and immunosuppression. In general, they are well-tolerated.

CQ has a strong antiviral effect in animal cells, in vitro. Though, the mechanism by which CQ decreases coronavirus infection is not unclear [38]. It appears that CQ raises the $\mathrm{pH}$ of endosomes, which prevents fusion and retards the virus from entering the cell. In addition, it may also block enzymes involved in the fusion between the virus and lung cells or thwart the viral replication process. CQ phosphate has demonstrated marked efficacy and acceptable safety in treating COVID-19 associated pneumonia in multicentre clinical trials conducted in China [39]. A combination of CQ with zinc has shown better effectivity. The ionized zinc inhibits the viral RNA polymerase and CQ acts as a zinc ionophore to rapidly transport zinc ions through cell membranes into cytoplasm.

HCQ shares the same mechanism of action as CQ, but its more tolerable safety profile makes it the preferred drug to treat malaria and autoimmune conditions. The optimal dose and duration of HCQ for treatment of COVID-19 are not well established. Various doses used by clinicians for HCQ are - 400 mg BID on day one, then once daily for 5 days; $400 \mathrm{mg}$ BID on day one, then $200 \mathrm{mg}$ BID for 4 days or $600 \mathrm{mg}$ BID on day one, then $400 \mathrm{mg}$ daily on days 2-5 [40].

The immunomodulatory effect of CQ as well as HCQ also may be useful in controlling the cytokine storm that occurs late-phase in critically ill SARS-CoV-2 infected patients [41]. HCQ has a superior in vitro anti-SARS-CoV-2 activity than CQ. In the study, this was demonstrated by the EC50 values for HCQ always being smaller than the EC50 values for CQ, indicating that HCQ has a more potent antiviral activity. Also, it may help mitigating the cytokine storm in critically ill SARSCoV-2 patients [42].

\section{Conclusion}

\section{Faux espoir, confusion and dormant options}

There are no specific FDA-approved drugs for the treatment of patients with COVID-19. At present the clinical management of COVID-19 includes infection prevention and control measures, and symptomatic and supportive care, including supplementary oxygen and mechanical respiratory support when indicated. A number of drugs approved for other indications as well as several investigational drugs are being studied in several clinical trials that are underway across the globe (Figure 3). In addition, several other drugs are being scanned for their likely role in COVID-19 prophylaxis or treatment in the United States and other countries [43].

Among the existing antiviral medicines, remdesivir is under clinical trials. There is evidence that it improves prognosis in COVID-19 patients. The convalescent plasma (CP) from patients recovered from the COVID-19 infection, contains antibodies against the virus and may be useful in supporting the immune system in severe cases. A recent study reported that the viral loads decreased to becoming undetectable in COVID-19 patients. The CP therapy was well tolerated with improvements in other clinical parameters ${ }^{44}$. The artificial antibodies against the virus, such as tocilizumab and Sarilumab, target interleukins like IL- 6 for treating the cytokine storms due to hyperactive immune response. In a small unpublished study in China, Actemra reduced pyrexia and requirement for supplemental oxygen [45].

The disease modifying and antiarthritic drugs against autoimmune diseases like rheumatoid arthritis work by dampening down the overactive immune system. They could be useful against in COVID-19 associated cytokine

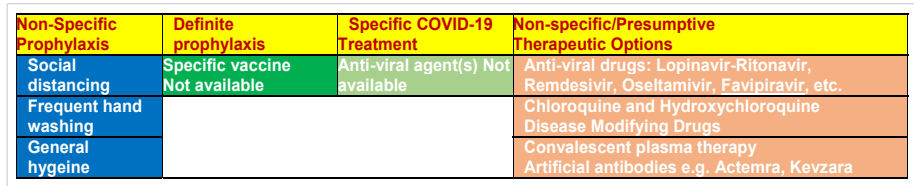

Figure 3: Present and Future Prophylactic and Therapeutic Options for COVID-19. 
release syndrome (CRS). The treatment for less severe CRS is supportive, addressing the symptoms like fever, muscle pain, or fatigue. Moderate CRS requires oxygen therapy, IV fluids and anti-hypotensive agents to deal with the shock. For moderate to severe CRS, the use of immunosuppressive agents like corticosteroids and Tocilizumab, an anti-IL6 monoclonal antibody, have been used in some medical centers to treat severe CRS without a proved positive outcome. CQ exhibits an inhibitory effect on SARS-CoV-2 replication cycle. However, the clinical use of CQ may cause severe side effects. The related compound, HCQ exhibits an antiviral effect and likely to attenuate the severe progression of COVID-19 by inhibiting the cytokine storm syndrome (CSS) by suppressing $\mathrm{T}$ cell activation, which is frequently fatal [46].

There are vaccine programmes to use messenger RNA to make cells produce anti-viral antibodies leading to immunity. This approach, though, has never been used in a widely available vaccine. There are notable vaccine development programs by biotech Moderna and BioNTech working with Pfizer [47]. Though, there is no vaccine for preexposure prophylaxis or postexposure management. A vaccine for COVID-19 may come in near future.

There are no specific approved drugs for the treatment for the disease. Though, a number of drugs are being scanned and studied in several clinical trials for their likely role in COVID-19 prophylaxis or treatment. As obvious, not all clinical trials will be successful, but having so many efforts in progress, some may succeed and provide a positive solution. Right now, though, confusion and despair prevail. The future seems afflicted with dormant therapeutic options as well as faux Espoir or false hopes. But, having so many efforts in progress, some may succeed and provide a positive solution. Right now, though, confusion and despair prevail.

\section{References}

1. Naming the coronavirus disease (COVID-19) and the virus that causes it. 2020. https://www.who.int/emergencies/diseases/novelcoronavirus-2019/technical-guidance/naming-the-coronavirusdisease-(covid-2019)-and-the-virus-that-causes-it

2. Lu R, Zhao X, Li J, Niu P, Yang B, et al. Genomic characterization and epidemiology of 2019 novel coronavirus: implications for virus origins and receptor binding. Lancet. 2020; 395: 565-574.

PubMed: https://www.ncbi.nlm.nih.gov/pubmed/32007145

3. Chan JF, Kok KH, Zhu Z, Chu H, To KK, et al. Genomic characterization of the 2019 novel human-pathogenic coronavirus isolated from a patient with atypical pneumonia after visiting Wuhan. Emerg Microbes Infect. 2020; 9: 221-236.

PubMed: https://www.ncbi.nlm.nih.gov/pubmed/31987001

4. Fehr AR, Perlman S. Coronaviruses: an overview of their replication and pathogenesis. Methods Mol Biol. 2015; 1282: 1-23.

PubMed: https://www.ncbi.nlm.nih.gov/pubmed/25720466

5. Li Q, Guan X, Wu P, Wang X, Zhou L, et al. Early Transmission Dynamics in Wuhan, China, of Novel Coronavirus-Infected Pneumonia. N Engl J Med. 2020; 382: 1199-1207.

PubMed: https://www.ncbi.nlm.nih.gov/pubmed/31995857
6. Cascella M, Rajnik M, Cuomo A, Dulebohn SC, Di Napoli R. et al. Features, Evaluation and Treatment Coronavirus (COVID-19). StatPearls Publishing LLC. Bookshelf ID: NBK554776. 2020; PMID: 32150360.

PubMed: https://www.ncbi.nlm.nih.gov/books/NBK554776/

7. Porter DL, Maloney DG. Cytokine release syndrome (CRS). UpToDate 2020. Retrieved from https://www.uptodate.com/contents/cytokinerelease-syndrome-crs?

8. Jose RJ, Manuel A. COVID-19 cytokine storm: the interplay between inflammation and coagulation. Lancet Resp Med. Corres. 2020. PubMed: https://www.ncbi.nlm.nih.gov/pubmed/32353251

9. Angeletti $S$, Benvenuto $D$, Bianchi M, Giovanetti $M$, Pascarella $S$, et al. COVID-2019: The role of the nsp2 and nsp3 in its pathogenesis. J Med Virol. 2020.

10. Lei J, Kusov $Y$, Hilgenfeld R. Nsp3 of coronaviruses: Structures and functions of a large multi-domain protein. Antiviral Res. 2018; 149: 58-74. PubMed: https://www.ncbi.nlm.nih.gov/pubmed/29128390

11. Tai $\mathrm{W}, \mathrm{He} \mathrm{L}$, Zhang $\mathrm{X}, \mathrm{Pu} J$, Voronin $\mathrm{D}$, et al. Characterization of the receptor-binding domain (RBD) of 2019 novel coronavirus: implication for development of RBD protein as a viral attachment inhibitor and vaccine. Cell Mol Immunol. 2020.

PubMed: https://www.ncbi.nlm.nih.gov/pubmed/32203189

12. Xu X, Chen $\mathrm{P}$, Wang J, Feng J, Zhou H, et al. Evolution of the novel coronavirus from the ongoing Wuhan outbreak and modelling of its spike protein for risk of human transmission. Sci China Life Sci. 2020; 63: 457-460.

PubMed: https://www.ncbi.nlm.nih.gov/pubmed/32009228

13. Chen Y, Liu Q, Guo D. Emerging coronaviruses: genome structure, replication, and pathogenesis. J Med Virol. 2020; 92: 418-423. PubMed: https://www.ncbi.nlm.nih.gov/pubmed/31967327

14. Kuba K, Imai Y, Rao S, Gao H, Guo F, et al. A crucial role of angiotensin converting enzyme 2 (ACE2) in SARS coronavirus-induced lung injury. Nat Med. 2020; 11: 875-879.

PubMed: https://www.ncbi.nlm.nih.gov/pubmed/16007097

15. Xu H, Zhong L, Deng J, Peng J, Dan H, et al. High expression of ACE2 receptor of 2019-nCoV on the epithelial cells of oral mucosa. Int J Oral Sci. 2020; $12: 8$.

16. In the author's opinion - inferring on the available findings and research data.

17. Zhao $Y$, Zhao Z, Wang $Y$, Zhou $Y$, Ma $Y$, et al. A novel coronavirus from patients with pneumonia in China. N Engl J Med. 2019. https://www. nejm.org/doi/full/10.1056/NEJMoa2001017

18. Zhao $Y$, Zhao Z, Wang $Y$, Zhou $Y$, Ma $Y$,et al. Single-cell RNA expression profiling of ACE2, the receptor of SARS-CoV-2. 2020.

19. Cao Y, Li L, Feng Z, Wan S, Huang $P$, et al. Comparative genetic analysis of the novel coronavirus (2019-nCoV/SARS-CoV-2) receptor ACE2 in different populations. Cell Discov. 2020; 6: 11.

PubMed: https://www.ncbi.nlm.nih.gov/pubmed/32133153

20. Guan WJ, Ni Z, Hu Y, Liang W, Ou C, et al. Clinical characteristics of 2019 novel coronavirus infection in China. N England J of Med. www. medrxiv.org/content/10.1101/2020.02.06.20020974v1

21. Huang C, Wang Y, Li X, Ren 4, Zhao J, et al. Clinical features of patients infected with 2019 novel coronavirus in Wuhan, China. Lancet. 2020; 395: 497-506.

PubMed: https://www.ncbi.nlm.nih.gov/pubmed/31986264

22. In the author's opinion - inferring on the available findings and research data. 
23. Anosmia, hyposmia and dysgeusia in the absence of other relevant disease should alert to the possibility of COVID-19 infection and warrant consideration for testing. American Academy of Otolaryngology-Head and Neck Surgery - position statement.

24. Tian $\mathrm{Y}$, Rong L, Nian $\mathrm{W}, \mathrm{He} \mathrm{Y}$. Review article: gastrointestinal features in COVID-19 and the possibility of faecal transmission. Aliment Pharmacol Ther. 2020; 51: 843-851.

PubMed: https://www.ncbi.nlm.nih.gov/pubmed/32222988

25. Wu Z, McGoogan JM. Characteristics of and Important Lessons from the Coronavirus Disease 2019 (COVID-19) Outbreak in China: Summary of a Report of 72314 Cases from the Chinese Center for Disease Control and Prevention. JAMA. 2020.

PubMed: https://www.ncbi.nlm.nih.gov/pubmed/32091533

26. Lianhan S, Jianping Z, Yi H, Du Ronghui, Bin C. On the use of corticosteroids for 2019-nCoV pneumonia. Lancet. 2020.

27. Baden LR, Rubin EJ. Covid-19 - The Search for Effective Therapy. 2020. PubMed: https://www.ncbi.nlm.nih.gov/pubmed/32187463

28. Cao B, Wang Y, Wen D, Liu W, Wang J, et al. A Trial of LopinavirRitonavir in Adults Hospitalized with Severe Covid-19. N Engl J Med. PubMed: https://www.ncbi.nlm.nih.gov/pubmed/32187464

29. Huang J. Efficacy of Chloroquine and Lopinavir/ Ritonavir in mild/ general novel coronavirus (CoVID-19) infections: a prospective, openlabel, multicenter randomized controlled clinical study. 2020. http://www.chictr.org.cn/showproj.aspx?proj=49263

30. https://www.the-scientist.com/news-opinion/flu-and-anti-hiv-drugsshow-efficacy-against-coronavirus-67052

31. Chen $C$, Zhang $Y$, Huang J, Yin $P$, Cheng Z, et al. Favipiravir versus Arbidol for COVID-19: A Randomized Clinical Trial. 2020. www. medrxiv.org/content/10.1101/2020.03.17.20037432v1

32. www.precisionvaccinations.com/avigan-favipiravir-t-705-broadspectrum-inhibitor-viral-rna-polymerase

33. Gordon CJ, Tchesnokov EP, Feng JY, Porter DP, Götte M. The antiviral compound remdesivir potently inhibits RNA-dependent RNA polymerase from Middle East respiratory syndrome coronavirus. J Biol Chem. 2020; 295: 4773-4779.

PubMed: https://www.ncbi.nlm.nih.gov/pubmed/32094225

34. Wang M, Cao R, Zhang L, Yang X, Liu J, et al. Remdesivir and chloroquine effectively inhibit the recently emerged novel coronavirus (2019-nCoV) in vitro. Cell Res. 2020; 30: 269-271.

PubMed: https://www.ncbi.nlm.nih.gov/pubmed/32020029

35. Gautret P, Lagier JC, Parola P, Hoang VT, Meddeb L, et al. Hydroxychloroquine and azithromycin as a treatment of COVID-19: results of an open-label non-randomized clinical trial. Int J Antimicrob
Agents. 2020; 105949.

PubMed: https://www.ncbi.nlm.nih.gov/pubmed/32205204

36. Liu J, Cao R, Xu M, Wang X, Zhang $\mathrm{H}$, et al. Hydroxychloroquine, a less toxic derivative of chloroquine, is effective in inhibiting SARSCoV-2 infection in vitro. Cell Discov. 2020; 6: 16.

PubMed: https://www.ncbi.nlm.nih.gov/pubmed/32194981

37. Colson P, Rolain JM, Lagier JC, Brouqui P, Raoult D, et al. Chloroquine and hydroxychloroquine as available weapons to Figureht COVID-19. Int J Antimicrob Agents. 2020; 105932.

PubMed: https://www.ncbi.nlm.nih.gov/pubmed/32145363

38. Vincent MJ, Bergeron E, Benjannet S, Erickson BR, Rollin PE, et al. Chloroquine is a potent inhibitor of SARS coronavirus infection and spread. Virol J. 2005; 2: 69.

PubMed: https://www.ncbi.nlm.nih.gov/pubmed/16115318

39. Gao J, Tian Z, Yang X. Breakthrough: Chloroquine phosphate has shown apparent efficacy in treatment of COVID-19 associated pneumonia in clinical studies. Biosci Trends. 2020; 14: 72-73. PubMed: https://www.ncbi.nlm.nih.gov/pubmed/32074550

40. Yao X, Ye F, Zhang M, Cui C, Huang B, et al. in vitro Antiviral Activity and Projection of Optimized Dosing Design of Hydroxychloroquine for the Treatment of Severe Acute Respiratory Syndrome Coronavirus 2 (SARS-CoV-2). Clin Infect Dis. 2020; pii: ciaa237.

PubMed: https://www.ncbi.nlm.nih.gov/pubmed/32150618

41. Keyaerts E, Vijgen L, Maes $P$, Neyts J, Van Ranst M. in vitro inhibition of severe acute respiratory syndrome coronavirus by chloroquine. Biochem Biophys Res Commun. 2004; 323: 264-268. PubMed: https://www.ncbi.nlm.nih.gov/pubmed/15351731

42. Yao X, Ye F, Zhang M, Cui C, Huang B, et al. in vitro Antiviral Activity and Projection of Optimized Dosing Design of Hydroxychloroquine for the Treatment of Severe Acute Respiratory Syndrome Coronavirus 2 (SARS-CoV-2). Clin Infect Dis. 2020; pii: ciaa237.

PubMed: https://www.ncbi.nlm.nih.gov/pubmed/32150618

43. Information on registered clinical trials for COVID-19 in the United States is available at: https://clinicaltrials.gov/external icon

44. Duan K, Liu B, Li C, Zhang H, Yu T, et al. The feasibility of convalescent plasma therapy in severe COVID-19 patients: a pilot study. 2020.

45. https://www.boston.com/news/health/2020/03/24/when-mightexperimental-drugs-to-treat-covid-19-be-ready

46. Zhou D, Dai SM, Tong Q. COVID-19: a recommendation to examine the effect of hydroxychloroquine in preventing infection and progression. $J$ Antimicrobial Chemotherapy. 2020; dkaa114.

47. www.cnbc.com/2020/03/17/hopes-of-a-coronavirus-vaccine-mountas-three-key-biotech-players-make-progress.html 